Urologe 2013 · 52:1055-1056

DOI 10.1007/s00120-013-3254-x

Online publiziert: 14. August 2013

(c) Springer-Verlag Berlin Heidelberg 2013

\author{
A. Schroeder ${ }^{1}$ M. Stöckle ${ }^{2}$ \\ ${ }^{1}$ Urologische Praxis, Neumünster \\ ${ }^{2}$ Klinik für Urologie und Kinderurologie, Universitätsklinikum Saarland, Homburg/Saar
}

\title{
Perspektiven zur Entwicklung der deutschen Urologie
}

lich Gelegenheit bieten, sich über den aktuellen Stand der Datenlage zu informieren und sich für künftige Diskussionen zu wappnen.

Vorschläge zur Umgestaltung des Gesundheitsmarktes gibt es reichlich. Sie kommen aus allen Ecken des Gesundheitswesens, der Politik und weiten Teilen der Gesellschaft. Eine zentrale Rolle nimmt dabei auch die fachärztliche Versorgung in Klinik und Praxis ein. Die bekannten Megatrends der gesellschaftlichen Alterung, einer steigenden und sich ändernden Gesundheitsnachfrage sowie eines wachsenden wirtschaftlichen Anpassungsdruckes werden Folgen für das Fach Urologie haben.

Innovative Arzneimittel und Technologie etwa sind Voraussetzungen für die Weiterentwicklung einer qualifizierten Patientenversorgung, der daraus resultierende Kostendruck wird sich aber auch in einer weiteren Verschärfung des Wettbewerbsdrucks niederschlagen

$\mathrm{Zu}$ einer Netzwerkmedizin gehören IV-Modelle, die vor diesem Hintergrund eine Renaissance erleben. Die Pharmaindustrie wird sich künftig an IV-Verträgen beteiligen. Für den BDU ist das Anlass, solchen Kooperationsmodellen offen gegenüber zu stehen und diese Form der Zusammenarbeit mit der Industrie vorzubereiten.

Klassische Sektorengrenzen von ambulanter und stationärer Versorgung verschieben sich bereits. Vormals stationäre Leistungen werden zunehmend ambulant erbracht. Gleichzeitig steigt die Zahl der Medizinischen Versorgungszentren unter Beteiligung von Krankenhäusern. Wirtschaftlicher Anpassungsdruck wird nicht nur die Konzentration des Klinikmarktes fördern. Auch Netze und Verbände werden sich stetig herausbilden, hin zu einer Netzwerkmedizin. Die ambulante spezialfachärztliche Versorgung (ASV) ist nur ein Beispiel dafür. Was in der ASV geht, was kommen kann, haben Christian Tschuschke und Holger Uhthoff skizziert.

Die Weiterbildungsordnung (WBO) ist deshalb nicht nur ein zentrales Element für strukturelle, praxisrelevante Weiterbildung zum Facharzt für Urologie. Sie ist auch von entscheidender Bedeutung für die Positionierung unseres Fachgebiets. Die jetzt von einer gemeinsamen Arbeitsgemeinschaft aus BDU und DGU vorbereitete Novellierung der MWBO (Musterweiterbildungsordnung) Urologie wird von Oliver Hakenberg und André Rollenhagen erläutert. Sie wird die Weichen für die Zukunft unseres Faches stellen.

Mit einer gemeinsamen Fort- und Weiterbildungsinstitution wie der Akademie der Deutschen Urologen ist ein Wissenstransfer im Fach Urologie gegeben, der den Weg in eine Wissensgesellschaft zu ermöglicht.

Erkenntnisgewinn, basierend auf Forschung, klinischen Studien ist eines. Die Erkenntnis muss aber auch tatsächlich in der Patientenversorgung ankommen. Damit bekommt Versorgungsforschung eine zunehmende Bedeutung und muss künftig in der Urologie umgesetzt werden. BDU und DGU bauen gemeinsam mit entsprechenden Arbeitskreisen und Sachausschüssen erste Strukturen dafür auf.

Die Versorgungsforschung steht in der Urologie am Anfang. Noch haben wir keine ausreichenden Antworten auf die Fragen „wer macht was in der urologischen ze der Prioritätenliste. Der kommende DGU-Kongress wird den Urologen reich- 
Patientenversorgung " wie es die Autoren Björn Volkmer, Alwin Weber und Wolfgang Bühmann in der Bestandsaufnahme 2013 zur Versorgungsforschung formulieren.

Wie Sie von unseren Autoren dieses Heftes erfahren, ist das Fach Urologie gut aufgestellt für künftige Anforderungen unseres Gesundheitssystems. Notwendige Prozesse des Wandels und der Anpassung betrachten wir nicht nur unter dem Aspekt der Risiken, sondern wir sehen besonders auch die Chancen, die sich darin in der Versorgung bieten. Wir Urologen sind willens, uns an der Gestaltung unverzichtbarer Anpassungsprozesse aktiv $\mathrm{zu}$ beteiligen.

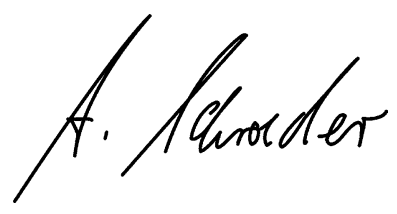

A. Schroeder

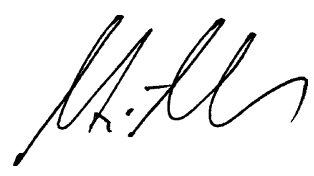

M. Stöckle
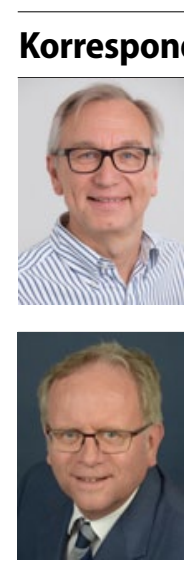

Prof. Dr. M. Stöckle

Klinik für Urologie und

Kinderurologie,

Universitätsklinikum Saarland,

Kirrberger Straße,

66421 Homburg/Saar

Michael.Stoeckle@

uniklinikum-saarland.de

Interessenkonflikt. A. Schroeder und M. Stöckle geben an, dass kein Interessenkonflikt besteht.

\section{Hodentumoren}

Obwohl bei Hodentumoren die Heilungschancen auf den ersten Blick sehr gut sind, bereiten Spätfolgen Schwierigkeiten und senken die Gesamtlebenserwartung der Patienten oft signifikant.

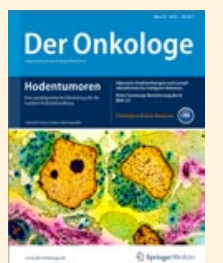

In der Ausgabe 7/2013 von Der Onkologe wird unter anderem diesen folgeschweren Begleiterkrankungen auf den Grund gegangen. Lesen Sie mehr zum Thema Hodentumoren in den Beiträgen

- Epidemiologie von Hodentumoren

- Seminom im klinischen Stadium I

- Nichtseminom im klinischen Stadium I

- Behandlung von Patienten mit cisplatinrefraktären Keimzelltumoren

- Stellenwert der Hochdosischemotherapie (HDCT) beim männlichen Keimzelltumor

- Spättoxizität und Survivorship bei Keimzelltumoren

Bestellen Sie diese Ausgabe zum Preis von 46,- Euro zzgl. Versandkosten bei Springer Customer Service Center Kundenservice Zeitschriften

Haberstr. 7

69126 Heidelberg

Tel.: +49 6221-345-4303

Fax: +49 6221-345-4229

E-Mail: leserservice@springer.com

Suchen Sie noch mehr zum Thema?

Mit e.Med, dem Online-Paket von Springer Medizin, können Sie schnell und komfortabel in über 500 medizinischen Fachzeitschriften recherchieren.

Weitere Infos unter springermedizin.de/ eMed. 